Supporting information to:

$\mathrm{H}_{2} \mathrm{O}_{2}$ in liquid fractions of hydrothermally pretreated biomasses: implications of lytic polysaccharide monooxygenases.

Riin Kont ${ }^{a}$, Ville Pihlajaniemi ${ }^{b}$, Klaus Niemelä ${ }^{b}$, Silja Kuuskª ${ }^{a}$ Kaisa Marjamaab ${ }^{b}$ Priit Väljamäe ${ }^{a^{*}}$

a Institute of Molecular and Cell Biology, University of Tartu, Riia 23b - 202, 51010 Tartu, Estonia.

b VTT, Technical Research Centre of Finland Ltd, P.O. Box 1000, 02044 VTT, Finland.

*Correspondence to: priit.valjamae@ut.ee

This supplementary material includes 11 pages, 2 tables, and 5 figures.

\title{
TABLE OF CONTENTS Page
}

$\begin{array}{ll}\text { Supplementary materials and methods } & \text { S2 }\end{array}$

Supplementary references $\quad$ S3

Table S1. Concentrations of $\mathrm{H}_{2} \mathrm{O}_{2}$ in liquid fractions (LF) measured at different S4 times of pre-incubation of LF.

Table S2. Changes in the concentration of the compounds in liquid fractions S5 - S6 upon aerobic incubation.

Figure S1. Titration of the liquid fractions (LFs) with $\mathrm{NaOH}$.

Figure S2. Measurement of $\mathrm{H}_{2} \mathrm{O}_{2}$ in the $96 \mathrm{~h}$ pre-incubated liquid fraction (LF) S8 of wheat straw.

Figure S3. Measurement of $\mathrm{H}_{2} \mathrm{O}_{2}$ in liquid fractions (LFs) using ferric-xylenol orange S9 (XO) assay.

Figure S4. The concentration of UV-phenolics in liquid fractions (LFs) at different S10 times of aerobic pre-incubation.

$\begin{array}{ll}\text { Modeling of oxygen supply and diffusion in the reaction system } & \mathrm{S} 10-\mathrm{S} 11\end{array}$

$\begin{array}{ll}\text { Figure S5. Modelling of oxygen concentration profiles. } & \text { S11 }\end{array}$ 


\section{Supplementary materials and methods}

\section{Measuring of $\mathrm{H}_{2} \mathrm{O}_{2}$ in liquid fractions using ferric-xylenol orange (XO) assay}

$60 \mu \mathrm{L}$ of $1.25 \mathrm{mM}$ xylenol orange (xylenol orange tetrasodium salt, Sigma-Aldrich 398187), and $30 \mu \mathrm{L}$ of $2.5 \mathrm{mM}$ ammonium ferrous sulfate (ammonium iron(II) sulfate hexahydrate, SigmaAldrich 215406) were added to $210 \mu \mathrm{L}$ of $\mathrm{H}_{2} \mathrm{O}_{2}$ (Honeywell, Lot\# SZBG2070) containing sample in 96-well microplate. After 30 min incubation in the dark at room temperature, the absorbance at $560 \mathrm{~nm}$ was measured using a microplate reader. All reagents, including the sample were made in $110 \mathrm{mM}$ perchloric acid. ${ }^{1,2}$ The stock solutions of the reagents were stored at $4{ }^{\circ} \mathrm{C}$ and used within a week. For measurement of $\mathrm{H}_{2} \mathrm{O}_{2}$ in liquid fractions (LFs) the standard curves of $\mathrm{H}_{2} \mathrm{O}_{2}(0$ $-20 \mu \mathrm{M}$ ) were made in the presence of suitably diluted LF. The concentration of $\mathrm{H}_{2} \mathrm{O}_{2}$ in LF was calculated from the intercept value of the absorbance using the slope measured at particular LF concentration. Before doing so the LF related, $\mathrm{H}_{2} \mathrm{O}_{2}$ independent absorbance, was subtracted from the intercept value of the absorbance. For measurement of the $\mathrm{H}_{2} \mathrm{O}_{2}$ independent absorbance, the $0.15 \mathrm{~mL}$ of LF was incubated with $0.1 \mu \mathrm{M}$ horseradish peroxidase before $\mathrm{XO}$ assay.

\section{Compositional analysis of liquid fractions}

Chromatographic analyses of monosaccharides in liquid fractions were made using DIONEX ICS5000 system equipped with CarboPac PA1 column and amperometric detection (HPAEC-PAD) as described in Tenkanen et al, $2000 .{ }^{3}$ For the total amount of carbohydrates the liquid fractions were treated with $3 \% \mathrm{H}_{2} \mathrm{SO}_{4}$ at $120{ }^{\circ} \mathrm{C}$ for $1 \mathrm{~h}$ before the analysis of monosaccharides with HPAEC-PAD. Concentration of furfural and 5-hydroxymethylfurfural was measured using Perkin Elmer Flexar HPLC system equipped with Aminex HPX-87 (Bio-Rad) column. Concentration of UV-phenolics was determined using absorbance of suitably diluted LFs at 215 and $280 \mathrm{~nm}$ according to the previously described protocols. ${ }^{4,5}$ In one set of experiment the LFs were buffered to $\mathrm{pH} 5$ and pre-incubated at $50^{\circ} \mathrm{C}$ under aerobic conditions before the measurement of UVphenolics. The dry matter (DM) content of liquid and solid samples was determined gravimetrically by oven-drying at $105^{\circ} \mathrm{C}$ overnight.

\section{Gas chromatography mass spectrometry (GC/MS) analyses}

Two sets of LFs, termed $\mathrm{N}_{2}$-LF and aerobic-LF, were subjected to GC/MS analyses for monitoring potential changes in the concentration of selected low molar mass compounds. For aerobic LF the $1 \mathrm{~mL}$ of frozen original LF was thawed, briefly centrifuged and $0.8 \mathrm{~mL}$ of the supernatant was mixed with $0.2 \mathrm{~mL}$ of the buffering solution (see "aerobic pre-incubation of LFs" in the main article). Buffered LF was pre-incubated in the dark at $50{ }^{\circ} \mathrm{C}$ for $24 \mathrm{~h}$. After pre-incubation the LF was rapidly frozen using liquid nitrogen and freeze-dried. The $\mathrm{N}_{2}$-LF was prepared as the aerobic-LF but the LF was frozen immediately after the addition of buffering solution. The freeze-dried samples were readily dissolved in $0.2 \mathrm{~mL}$ of pyridine and trimethylsilylated with $0.2 \mathrm{~mL}$ of BSTFA (containing $10 \%$ of TMCS), after addition of $0.2 \mathrm{mg}$ salicylic acid as the internal standard. The GC/MS runs were performed with an Agilent 6890 series GC system, equipped with an Agilent 5973 mass selective detector and a DB-5 MS capillary column $(30 \mathrm{~m} \times 0.25 \mathrm{~mm}$, film thickness $0.25 \mu \mathrm{m}$ ). The applied temperature program was $1 \mathrm{~min}$ at $80^{\circ} \mathrm{C}, 10^{\circ} \mathrm{C} / \mathrm{min}$ to $300^{\circ} \mathrm{C}$, and $10 \mathrm{~min}$ at $300{ }^{\circ} \mathrm{C}$. The injection split ratio was $25: 1$. The MS identifications were based on the use of relevant literature ${ }^{6,7}$, in-house MS library, and commercial Wiley database. Indicative quantitative calculations were based on the peak areas without corrections. 


\section{Supplementary references}

1. Gay, C.; Collins, J.; Gebicki, J. M. Hydrogen peroxide assay with the ferric-xylenol orange complex. Anal. Biochem. 1999, 273, 149-155.

2. Gay, C.; Gebicki, J. M. Perchloric acid enhances sensitivity and reproducibility of the ferricxylenol orange peroxide assay. Anal. Biochem. 2002, 304, 42-46.

3. Tenkanen, M.; Siika-Aho, M. An a-glucuronidase of Schizophyllum commune acting on polymeric xylan. J. Biotechnol. 2000, 78, 149-161.

4. Goldschmid, O. Ultraviolet spectra, in: Sarkanen, K. V.; Ludwig, C. H. (Eds.), Lignins: Occurrence, Formation, Structure and Reactions. John Wiley \& Sons Inc., 1971, pp. $241-$ 298.

5. Sluiter, A.; Hames, B.; Ruiz, R. O.; Scarlata, C.; Sluiter, J.; Templeton, D.; Crocker, D. Determination of Structural Carbohydrates and Lignin in Biomass: Laboratory Analytical Procedure (LAP), Technical Report NREL/TP-510-42618. National Renewable Energy Laboratory, U.S. Department of Energy. 2011.

6. Borrega, M.; Niemela, K.; Sixta, H. Effect of hydrothermal treatment intensity on the formation of degradation products from birchwood. Holzforschung 2013, doi:10.1515/hf2013-0019.

7. Isidorov, V. A. GC-MS of biologically and environmentally significant organic compounds: TMS derivatives. 2020, John Wiley \& Sons, 706 pp. 
Table S1. Concentrations of $\mathrm{H}_{2} \mathrm{O}_{2}$ in liquid fractions (LF) measured at different times of preincubation of $\mathrm{LF}$ (at pH 5 and $50{ }^{\circ} \mathrm{C}$ ) measured using TrAA9A/ $/{ }^{14} \mathrm{C}-\mathrm{BMCC}(\mathrm{LPMO})$ or ferric-xylenol orange $(X O)$ method. Concentrations of $\mathrm{H}_{2} \mathrm{O}_{2}$ refer to the concentrations that have been extrapolated to those in original, $100 \%$ LF.

\begin{tabular}{|c|c|c|c|c|c|c|}
\hline \multirow{3}{*}{$\begin{array}{c}\text { Pre- } \\
\text { incubation } \\
\text { time }\end{array}$} & \multicolumn{6}{|c|}{ Concentration of $\mathrm{H}_{2} \mathrm{O}_{2}$ in $100 \% \mathrm{LF}(\mu \mathrm{M})$} \\
\hline & \multicolumn{2}{|c|}{ Wheat straw $A^{a}$} & \multicolumn{2}{|c|}{ Birch $A^{a}$} & \multicolumn{2}{|c|}{ Pine $A^{a}$} \\
\hline & LPMO & $\mathrm{XO}$ & LPMO & $\mathrm{XO}$ & LPMO & $\mathrm{XO}$ \\
\hline $0.5 \mathrm{~h}$ & $316 \pm 6$ & $88 \pm 23$ & $85 \pm 10$ & $25 \pm 10$ & $31 \pm 28$ & $10 \pm 2$ \\
\hline $24 \mathrm{~h}$ & $175 \pm 20$ & $128 \pm 10$ & $42 \pm 2$ & $44 \pm 5$ & $-1.5 \pm 5.2$ & $6.4 \pm 3.1$ \\
\hline $48 \mathrm{~h}$ & $402 \pm 19$ & $271 \pm 23$ & $74 \pm 9$ & $47 \pm 1$ & $3.8 \pm 1.3$ & $7.2 \pm 1.8$ \\
\hline \multirow[t]{5}{*}{$96 \mathrm{~h}$} & 704 & $725 \pm 151$ & $168 \pm 33$ & $160 \pm 4$ & $9.1 \pm 2.6$ & $9.5 \pm 1.5$ \\
\hline & \multicolumn{6}{|c|}{ Rate of $\mathrm{H}_{2} \mathrm{O}_{2}$ accumulation $\left(V_{\text {accumul }}, \mu \mathrm{M} \mathrm{h}^{-1}\right)$ in $100 \% \mathrm{LF}$} \\
\hline & \multicolumn{2}{|c|}{ Wheat straw A } & \multicolumn{2}{|c|}{ Birch A } & \multicolumn{2}{|c|}{ Pine A } \\
\hline & LPMO & $\mathrm{XO}$ & LPMO & $\mathrm{XO}$ & LPMO & $\mathrm{XO}$ \\
\hline & $8.4 \pm 1.4$ & $8.5 \pm 2.3$ & $1.78 \pm 0.48$ & $1.72 \pm 0.01$ & $0.14 \pm 0.1$ & $0.04 \pm 0.06$ \\
\hline
\end{tabular}

a Note that the results obtained using the LPMO method show the average values and S.D. of two experiments that include a single parallel of hydrothermal pre-treatment of biomass (parallel A) that was tested with 0.2 and $0.5 \mu \mathrm{M}$ TrAA9A in a single parallel. On the other hand, the data presented in the main article show the average values and S.D. of four experiments that include two parallels (parallels $A$ and $B$ ) of hydrothermal pre-treatment of biomass each tested with 0.2 and $0.5 \mu \mathrm{M}$ TrAA9A in a single parallel. 
Table S2. Changes in the concentration (Aerobic-LF minus $\mathrm{N}_{2}-\mathrm{LF}$ ) of the compounds in liquid fractions upon aerobic incubation at $\mathrm{pH} 5$ and $50^{\circ} \mathrm{C}$ for $24 \mathrm{~h}$. The concentrations were calculated from the amounts of the compounds listed in Table 3 of the main article. Concentrations (in $\mu \mathrm{M}$ ) refer to the concentrations in original $100 \%$ LF.

\begin{tabular}{l|c|c|c}
\hline \multirow{2}{*}{ Compounds and categories } & \multicolumn{3}{|c}{ Change in concentration (Aerobic-LF - N2-LF, $\mu \mathrm{M}$ ) } \\
\cline { 2 - 4 } & Wheat straw & Birch & Pine \\
\hline Simple phenols (H lignols) & -29 & 0 & 0 \\
4-Vinylphenol & 0 & -783 & 0 \\
4-Hydroxyacetophenone & -14 & 0 & 0 \\
1-(4-Hydroxyphenyl)ethanol & -13 & -39 & 0 \\
4-Hydroxybenzoic acid & 30 & 0 & 0 \\
p-Coumaric acid & & &
\end{tabular}

Guaiacyl compounds (G lignols)

Guaiacol

Vanillin

1-Guaiacylethanol

Vanillic acid

Dihydroconiferyl alcohol

Coniferyl alcohol

1-Hydroxy-3-guaiacylacetone, ketonic

1-Hydroxy-3-guaiacylacetone, enolic $\quad-22$

1-Guaiacylglycerols (2 isomers) 3

Ferulic acid $\quad-28$

Unknown guaiacyl compound

$-15$

\begin{tabular}{c|c|c}
-11 & 0 & 0 \\
3 & 0 & -12 \\
48 & 0 & 0 \\
-27 & 0 & 17 \\
0 & 0 & 94 \\
2 & 0 & 0 \\
0 & 0 & -82 \\
-22 & 0 & -12 \\
3 & 0 & -12 \\
-28 & 0 & 0 \\
-15 & 21 & 0 \\
\hline
\end{tabular}

Syringyl compounds (S lignols)

Syringol

Dihydrosinapyl alcohol

1-Syringylglycerols (2 isomers)

1-Hydroxy-3-syringylacetone, enolic

Benzenediols and benzenetriols

Catechol

Hydroquinone

2-Methylhydroquinone

Pyrogallol

1,2,4-Benzenetriol

\begin{tabular}{c|c|c}
8 & -75 & 0 \\
0 & -34 & 0 \\
-83 & -9 & 0 \\
2 & -138 & 0
\end{tabular}

Unknown phenols (monolignols)

Unknown phenol 1

Unknown phenol 2

Dilignols or lignans

Secoisolariciresinol

Syringaresinol

Total formed phenolics $(\mu \mathrm{M})$

\begin{tabular}{c|c}
1 & -18 \\
-31 & -56 \\
-29 & -37 \\
0 & -25 \\
-99 & 0
\end{tabular}

\begin{tabular}{c|c}
-18 & 0 \\
-56 & -13 \\
-37 & 0 \\
-25 & 0 \\
0 & 0
\end{tabular}

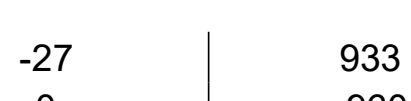
933
-960

690

0 0

\footnotetext{
Totalformed phenolics ( $\mu M)$
}

\begin{tabular}{|c|c} 
& 0 \\
& 13 \\
\hline ics $(\mu \mathrm{M})$ & 111
\end{tabular}

\begin{tabular}{c|r}
0 & 0 \\
13 & 6 \\
\hline 111 & 960
\end{tabular}

\begin{tabular}{c|c}
0 & 21 \\
6 & 0 \\
\hline 960 & 822
\end{tabular}




\begin{tabular}{l|c|c|c} 
Total lost phenolics $(\mu \mathrm{M})$ & 329 & 2174 & 132 \\
\hline Other compounds of interest & & & \\
Pyruvic acid & -795 & -505 & 70 \\
2-Oxobutanoic acid & -103 & 0 & 0 \\
Reductic acid & -463 & -245 & 0 \\
2-Furoic acid & -6 & 9 & 3 \\
5-Hydroxymethylfurfural & 0 & 0 & -272 \\
Malic acid & -152 & 251 & 0 \\
\hline Total formed $(\mu \mathrm{M})$ & 0 & 9 & 70 \\
Total lost $(\mu \mathrm{M})$ & 1518 & 749 & 272 \\
\hline
\end{tabular}




\section{Supplementary Figures}

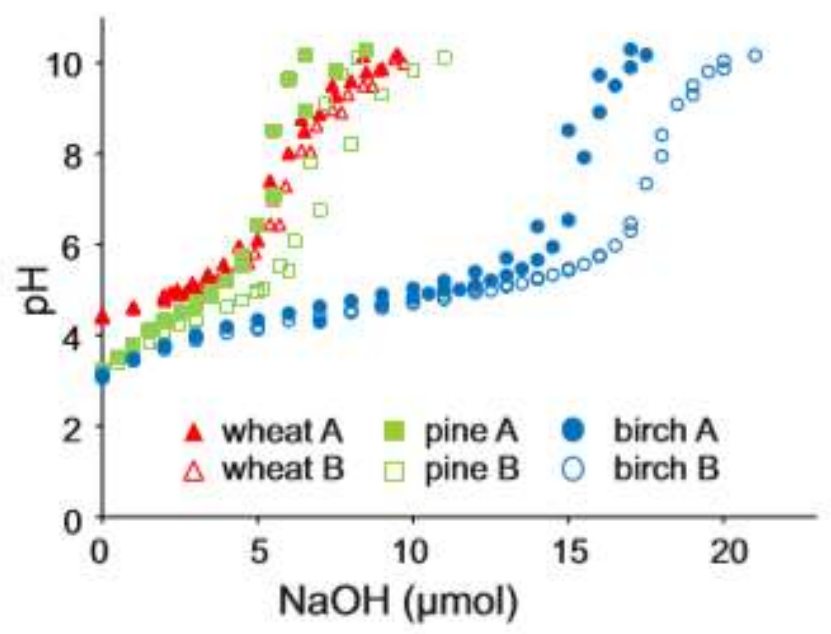

Figure S1. Titration of the liquid fractions (LFs) with $\mathrm{NaOH}$. Frozen LFs were thawed and 1.7 $\mathrm{mL}$ aliquots were titrated with $1.0 \mathrm{M} \mathrm{NaOH}$ in glass tubes at $50^{\circ} \mathrm{C}$. Shown are the overlaid results of two independent titrations. Designations A and B refer to two independent parallels of hydrothermal pre-treatment. 


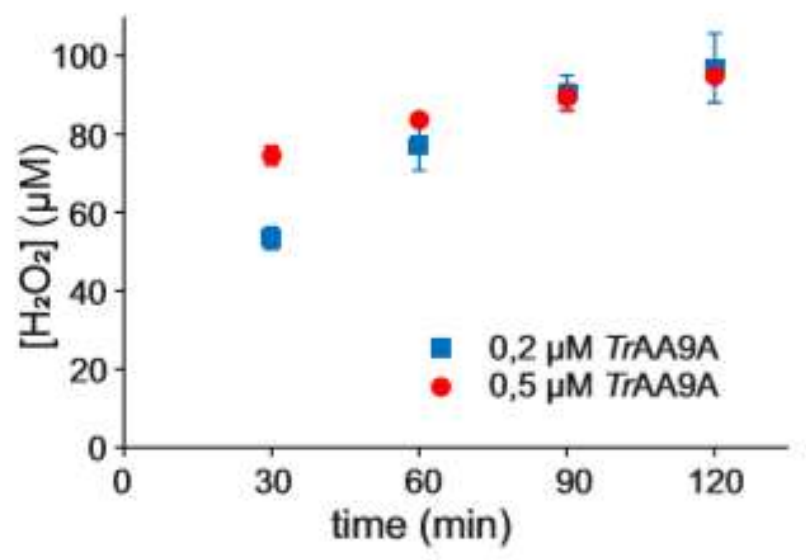

Figure S2. Measurement of $\mathrm{H}_{2} \mathrm{O}_{2}$ in the $96 \mathrm{~h}$ pre-incubated liquid fraction (LF) of wheat straw. The reactions were made in $50 \mathrm{mM}$ sodium acetate $\mathrm{pH} 5.0$ at $50^{\circ} \mathrm{C}$. The concentration of ${ }^{14} \mathrm{C}$-BMCC was $0.6 \mathrm{~g} \mathrm{~L}^{-1}$ and that of the LF was $10 \%(\mathrm{v} / \mathrm{v})$. The concentration of TrAA9A was either 0.2 or $0.5 \mu \mathrm{M}$ (as indicated in the plot). Before experiment the LF was pre-incubated at pH 5 and $50^{\circ} \mathrm{C}$ for $96 \mathrm{~h}$. Shown are the average values of two experiments, one made with the wheat straw $A$ and the other with the wheat straw $B$ (i.e. two parallels of hydrothermal pre-treatment of wheat straw). Error bars show S.D. 

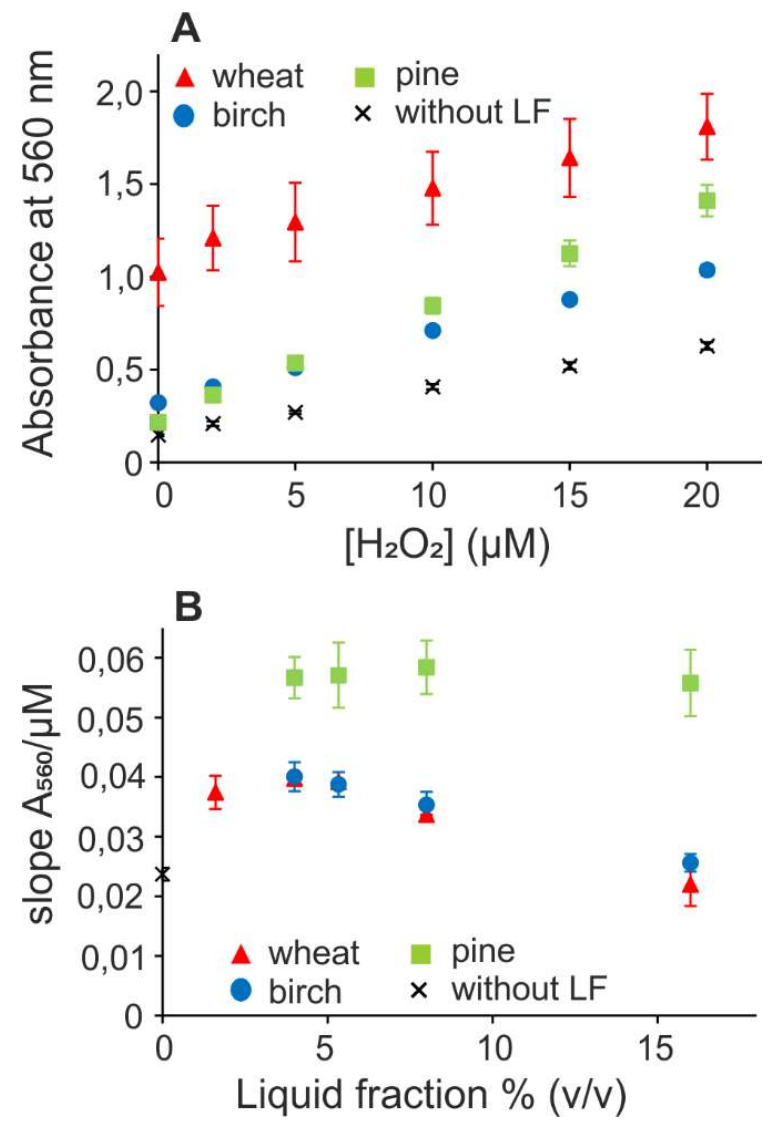

Figure S3. Measurement of $\mathrm{H}_{2} \mathrm{O}_{2}$ in liquid fractions (LFs) using ferric-xylenol orange (XO) assay. (A) Standard curves of $\mathrm{H}_{2} \mathrm{O}_{2}$ in $\mathrm{XO}$ assay made in the absence and presence of $8 \%(\mathrm{v} / \mathrm{v}) \mathrm{LF}$. Before XO assay, the LF was pre-incubated at $\mathrm{pH} 5$ and $50^{\circ} \mathrm{C}$ for $48 \mathrm{~h}$. Solid lines show linear regression of the data. (B) Dependency of the slope of the standard curve) on the type and the concentration of LF. Before XO assay, the LF was pre-incubated at $\mathrm{pH} 5$ and $50^{\circ} \mathrm{C}$ for $48 \mathrm{~h}$. 


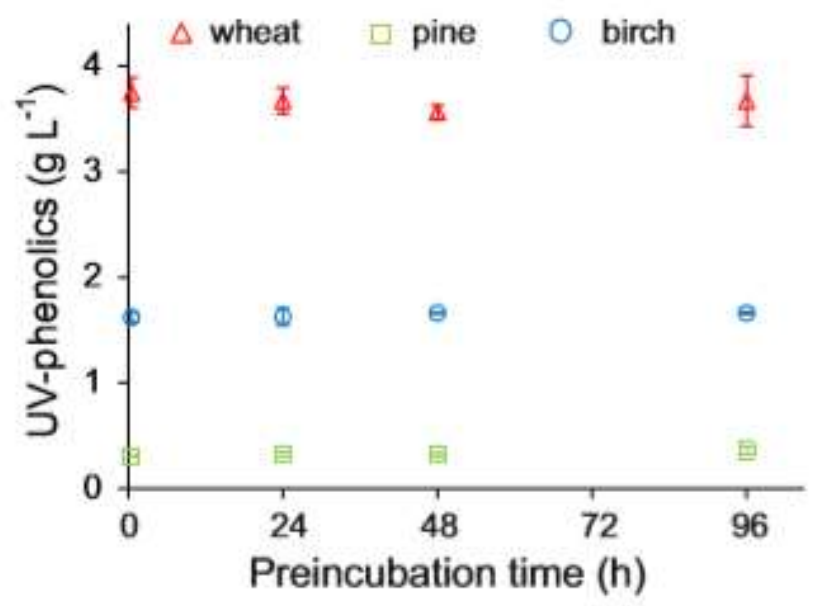

Figure S4. The concentration of UV-phenolics in liquid fractions (LFs) at different times of aerobic pre-incubation. Before the measurement of the concentration of UV-phenolics the LFs were pre-incubated at $\mathrm{pH} 5$ and $50^{\circ} \mathrm{C}$ for $0.5-96 \mathrm{~h}$. Shown are the average values and S.D. of two experiments made using a single parallel of the hydrothermal pre-treatment of biomass (wheat straw $A$, birch $A$, and pine $A$ ).

\section{Modeling of oxygen supply and diffusion in the reaction system}

During pre-incubation experiments, the amount of oxygen in the $\sim 0.5 \mathrm{~mL}$ of air in the vial headspace was $19 \mu \mathrm{mol}$, which is in excess of the highest observed daily $\mathrm{H}_{2} \mathrm{O}_{2}$ formation of $\sim 3200$ $\mu M$, or $3.2 \mu \mathrm{mol}$ in the $1 \mathrm{~mL}$ reaction volume (Figure $2 \mathrm{C}$ ).

In order to assess whether oxygen diffusion could limit the reaction rate in $\mathrm{H}_{2} \mathrm{O}_{2}$ determination, a simplified diffusion model was applied for testing whether diffusion could sustain the observed reaction rates continuously, i.e. in steady state. Given the substantially higher diffusion rate in gas phase, the concentration gradient in gas phase was assumed negligible and saturation concentration ( $\left.c_{s a t}\right)$ of $\mathrm{O}_{2}$, according to Henry's law (Eq. S1), was assumed on the liquid surface.

$$
c_{\text {sat }}=k_{H} p_{O 2}=177.5 \mu \mathrm{M}
$$

where $k_{H, 500^{\circ} \mathrm{C}}=0.000847 \mathrm{~mol} \mathrm{~L}^{-1} \mathrm{bar}^{-1}$ is the Henry's constant for $\mathrm{O}_{2}$ at $50{ }^{\circ} \mathrm{C}$, and partial pressure of oxygen is $p_{O 2}=0.2095 \mathrm{bar}$. Diffusion of $\mathrm{O}_{2}$ in the liquid phase was numerically solved in one dimension. A liquid phase of a depth of $2 \mathrm{~cm}$ was discretized into $n=1000$ equal slices, and a finite difference approximation of Fick's $2^{\text {nd }}$ law of diffusion for each slice $i$ was applied as:

$$
\frac{\partial c}{\partial t}=D \frac{\partial^{2} c}{\partial x^{2}}-R \approx D \frac{c_{i-1}-c_{i}+c_{i+1}}{(\Delta x)^{2}}-R
$$

A diffusion coefficient $\mathrm{D}$ of $3.99 * 10^{-5} \mathrm{~cm}^{2} \mathrm{~s}^{-1}$ was applied for $\mathrm{O}_{2}$ in water at $50{ }^{\circ} \mathrm{C}$. Boundary conditions $c_{1}=c_{s a t}$ for surface and $c_{n+1}=c_{n}$ for bottom were applied, and reactive oxygen consumption was included, assuming pseudo-first order rate, $R=k c_{i}$. The time derivative of concentration was solved for each slice numerically, using the ode $15 \mathrm{~s}$ differential equation solver of Matlab R2020b (Mathworks). Steady state was expected to be reached after $100 \mathrm{~h}$ of reaction 
time. Reaction rate was calculated as the average rate along the depth for steady state, and the average in depth and time for a fixed reaction time.

Hypothetical pseudo-first order reaction constants for $\mathrm{O}_{2}$ consumption that would lead to overall steady state reaction rates equal to the apparent observed maximum $\left(63 \mu \mathrm{M} \mathrm{h}^{-1}\right)$ and minimum $\left(4.5 \mu M h^{-1}\right)$ rates of $\mathrm{H}_{2} \mathrm{O}_{2}$ formation (Fig. $2 \mathrm{~A}$ ) were iteratively found to be $k_{63} \mu \mathrm{M} h^{-1}$, steady state $=$ $2.46 * 10^{-4} \mathrm{~s}^{-1}$ and $k_{4.5 \mathrm{M} h^{-1} \text {, steady state }}=3.98 * 10^{-6} \mathrm{~s}^{-1}$. These reaction constants led to steady state concentration profiles shown in Figure S5, showing $20 \%$ and $89 \%$ overall $\mathrm{O}_{2}$ saturation, respectively. Diffusion of $\mathrm{O}_{2}$ is therefore sufficient to sustain both reaction rates indefinitely, although the rates are likely affected by the degree of saturation. The concentration profile after the at the maximum rate after the 30 min time interval in $\mathrm{H}_{2} \mathrm{O}_{2}$ determination was also modelled, assuming $80 \% \mathrm{O}_{2}$ saturation after mixing during sampling. The reaction constant matching the observed $63 \mu M h^{-1}$ in $0.5 \mathrm{~h}$ was $k_{63 \mu M h^{-1}, 0.5 h}=9.8 * 10^{-5} \mathrm{~s}^{-1}$, and the corresponding $\mathrm{O}_{2}$ saturation was $71 \%$, showing that the initial aeration alleviated the effect of diffusion at high reaction rates.

In conclusion, the modelling results imply that although oxygen diffusion is likely a contributing factor in the determined reaction rates, it is not rate limiting.

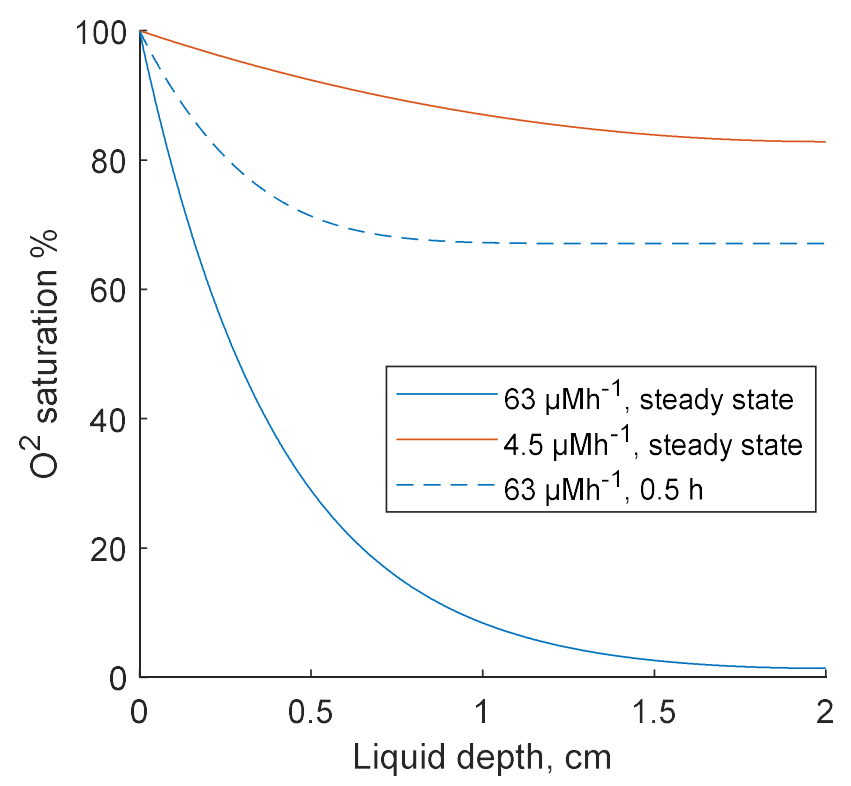

Figure S5. Modelling of oxygen concentration profiles in the $\mathrm{H}_{2} \mathrm{O}_{2}$ determination at steady-state reaction rates of 63 and $4.5 \mu M h^{-1}$, and at an average reaction rate of $63 \mu M h^{-1}$ within a $30 \mathrm{~min}$ interval with initial $80 \%$ oxygen saturation. 